Document downloaded from:

http://hdl.handle.net/10251/151051

This paper must be cited as:

Fuente, D.; Lizama, C.; Urchueguía Schölzel, JF.; Conejero, JA. (2018). Estimation of the light field inside photosynthetic microorganism cultures through Mittag-Leffler functions at depleted light conditions. Journal of Quantitative Spectroscopy and Radiative Transfer. 204:23-26. https://doi.org/10.1016/j.jqsrt.2017.08.012

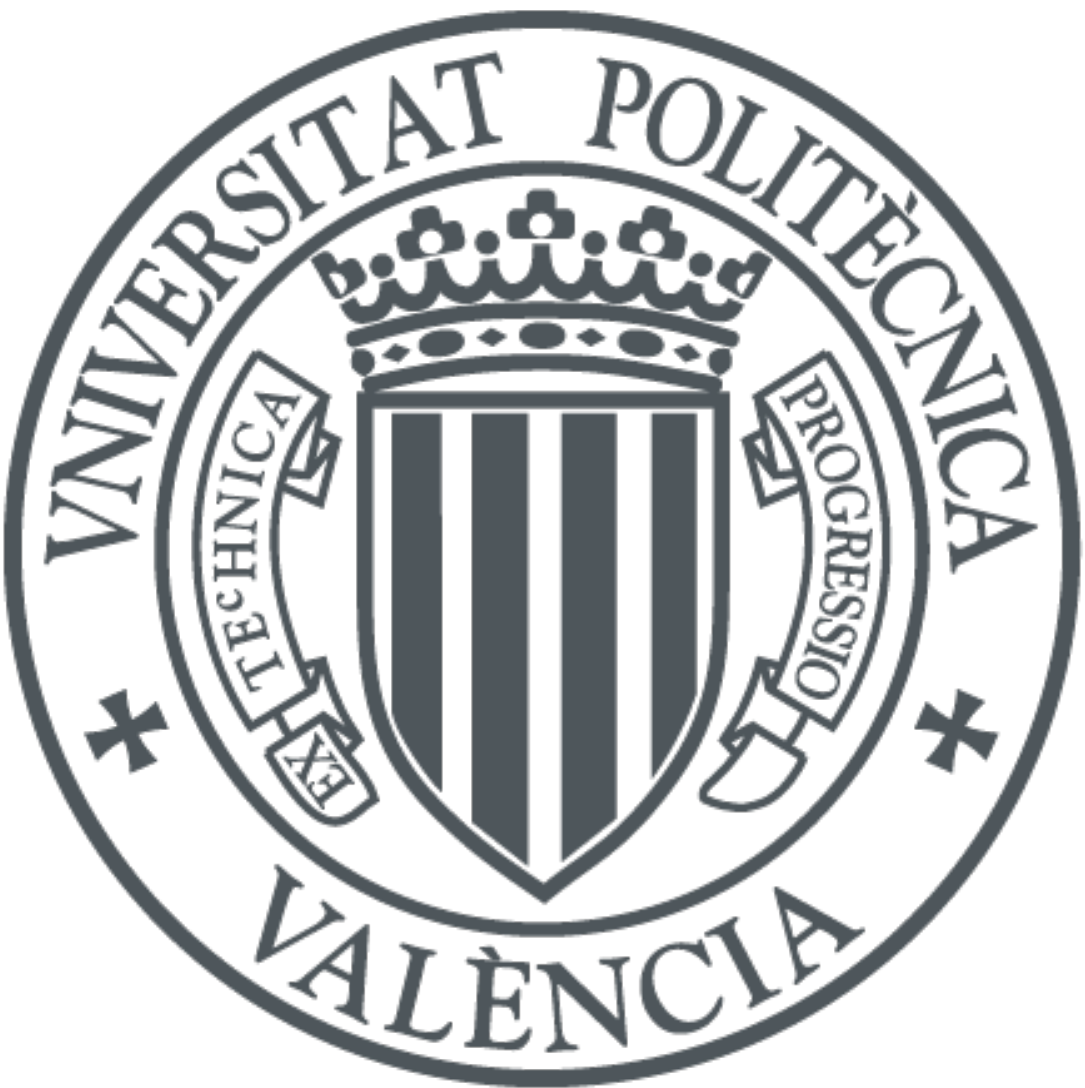

The final publication is available at

https://doi.org/10.1016/j.jqsrt.2017.08.012

Copyright Elsevier

Additional Information 


\title{
Estimation of the light field inside photosynthetic microorganism cultures through Mittag-Leffler functions at depleted light conditions
}

\author{
David Fuente ${ }^{1, *}$, Carlos Lizama ${ }^{2}$, Javier F. Urchueguía ${ }^{1}$, and J. Alberto Conejero ${ }^{3}$ \\ ${ }^{1}$ Instituto de Aplicaciones de las Tecnologías de la Información y de las Comunicaciones Avanzadas, \\ Universitat Politècnica de València, València, Spain \\ ${ }^{2}$ Universidad de Santiago de Chile, Facultad de Ciencia, Departamento de Matemática y Ciencia de \\ la Computación, Santiago de Chile, Chile \\ ${ }^{3}$ Instituto Universitario de Matemática Pura y Aplicada, Universitat Politècnica de València, \\ València, Spain
}

September 4, 2017

\begin{abstract}
Light attenuation within suspensions of photosynthetic microorganisms has been widely described by the Lambert-Beer equation. However, at depths where most of the light has been absorbed by the cells, light decay deviates from the exponential behaviour and shows a lower attenuation than the corresponding from the purely exponential fall. This discrepancy can be modelled through the Mittag-Leffler function, extending Lambert-Beer law via a tuning parameter $\alpha$ that takes into account the attenuation process.

In this work, we describe a fractional Lambert-Beer law to estimate light attenuation within cultures of model organism Synechocystis sp. PCC 6803. Indeed, we benchmark the measured light field inside cultures of two different Synechocystis strains, namely the wild-type and the antenna mutant strain called Olive at five different cell densities, with our in silico results. The Mittag-Leffler hyper-parameter $\alpha$ that best fits the data is 0.995 , close to the exponential case. One of the most striking results to emerge from this work is that unlike prior literature on the subject, this one provides experimental evidence on the validity of fractional calculus for determining the light field.

We show that by applying the fractional Lambert-Beer law for describing light attenuation, we are able to properly model light decay in photosynthetic microorganisms suspensions.
\end{abstract}

Keywords: Light field; attenuation; Lambert-Beer law; Mittag-Leffler; modelling; Synechocystis

\section{Introduction}

Photosynthetic microorganisms are capable of absorbing light by means of a wide variety of photo-active pigments, whose active molecules can get excited at given wavelengths. Photosynthetic pigments cover the so called Photosynthetically Active Radiation (PAR), comprising the wavelength range of 400 to $700 \mathrm{~nm}$, which practically overlaps the visible spectrum for human beings.

Light decay in photosynthetic cultures is due to absorption and scattering phenonema [1], can be described using inherent optical properties of the suspension [2] and by solving a self-consistency problem between average irradiance inside the whole volume and the average PAR downward attenuation coefficient, as both magnitudes depend on each other. In this regard, a modelling approach was recently proposed [3], where the remaining light given as a PAR integrated irradiance value at different depths from the emission lamp within Synechocystis sp. PCC 6803 (hereafter referred to as Synechocystis) cultures was in silico estimated. 
However, the model employs the Bouguer law, postulated in Bouguer's Essai d'Optique in 1729 [4] and commonly known in Optics as Lambert-Beer law, for estimating remaining irradiance up on depth and hence it fails in the evaluation of very low light intensities. Despite light usually follows an exponential decay that can be described by the Lambert-Beer law, it is known that at low illumination conditions, below the so called euphotic zone, light attenuation deviates from a purely exponential behaviour inside biological cultures. As a rule of thumb, the exponential case is valid within the interval comprised between that layer of water from the surface down to the depth at which downward irradiance is $1 \%$ of that at the depth $z=0$ [5].

This has been reported in several works on oceanic environments $[6,7]$ but also in photosynthetic microorganism cultures grown in artificial conditions [8]. To overcome the poor attenuation prediction capabilities of current models near or within the euphotic zone, the algorithms can be modified to extend exponential attenuation to a more complex representation by means of Mittag-Leffler (ML) functions. These are solutions of fractional order integral and fractional order differential equations and have been successfully introduced in several applied areas for studying different problems such as rheology [9], diffusion in porous solids [10], superdiffusive transport and Lévy motion [11], protein ligand rebinding [12], electrical networks [13] and, in general, they are useful to study relaxation phenomena in complex physical systems [14, 15] and in probability theory [16]. Moreover, it is remarkable that ordinary and generalised Mittag-Leffler functions interpolate between a purely exponential law and a power-like behaviour of phenomena governed by ordinary kinetic equations and their fractional counterparts [17, 18, 19].

Regarding optical applications with such class of mathematical functions, in [20] a ML function for the modulation of a Gaussian beam was employed and the splitting and accelerating properties of the obtained Mittag-Leffler-Gaussian beam described. Recently, the light attenuation modelling through a generalisation of the exponential function via Mittag-Leffler functions was considered [21]. That generalisation of the Lambert-Beer rises the accuracy of the predictions and explains possible deviations from the exponential extinction law in spatially correlated media. This model was later improved by introducing a new formal description that included Wright type functions to express light transmission probability in random media [22]. Estimations of the deviation from the exponential law were also exposed. In this way, multi-order fractional operators (integral and differential) in the unit disk have been also used for extending the LambertBeer equation [23].

Nevertheless, in none of these works experimental data were assessed. In summary, we have considered the fractional generalisation of the Lambert-Beer law and modelled light attenuation within cultures of model organism Synechocystis sp. PCC 6803. An interesting outcome of this research is that all experimental measurements are properly modelled by just fitting the hyper-parameter $\alpha$ of the ML functions. Indeed, just one value is needed for both studied Synechocystis strains, namely the wild-type and the antenna mutant strain called Olive at all, i.e. five different, cell densities. These results back the use of fractional calculus for describing light attenuation in photobioreactors (PBRs).

\section{Methodology}

Mittag-Leffler (ML) functions, introduced by the homonym Swedish mathematician Gösta Mittag-Leffler, belong to a family of special functions characterised by two complex parameters $\alpha$ and $\beta$. When the real part of $\alpha$ is strictly positive, they can be defined by means of the following convergent series:

$$
E_{\alpha}(z)=\sum_{k=0}^{\infty} \frac{z^{k}}{\Gamma(1+\alpha k)}, \quad \alpha \in \mathbb{C}, \quad \Re(\alpha)>0, \quad z \in \mathbb{C},
$$

or in its more general form by

$$
E_{\alpha, \beta}(z)=\sum_{k=0}^{\infty} \frac{z^{k}}{\Gamma(\beta+\alpha k)}, \quad \alpha, \beta \in \mathbb{C}, \quad \Re(\alpha)>0, \quad \Re(\beta)>0, \quad z \in \mathbb{C} .
$$


The positivity of the real parts of $\alpha$ and $\beta$ yields that they become entire functions. Here, $\Gamma(\cdot)$ stands for the Gamma function of Euler, that generalises the factorial, since $\Gamma(n):=(n-1)$ ! for every natural number $n \in \mathbb{N}$. For every $z \in \mathbb{C}$ with $\Re(z)>0$, this function can be defined by the following convergent improper integral

$$
\Gamma(z):=\int_{0}^{\infty} t^{z-1} e^{-t} d t
$$

ML functions generalise the exponential functions. In particular, if $\alpha=\beta=1$, we get the series of the exponential function. They arise as solutions of the following abstract Cauchy problem

$$
\left\{\begin{array}{l}
\left(\frac{d}{d x}\right)^{\alpha} f(x)=-\lambda f(x), \quad x>0 \\
f(0) \in \mathbb{R} \text { given }
\end{array}\right.
$$

where $\left(\frac{\mathrm{d}}{\mathrm{dx}}\right)^{\alpha}$ stands for the Caputo fractional derivative of real order $\alpha$ respect to $x$. As an easy way to understand what is a fractional derivative, one can think that $\left(\frac{d}{d x}\right)^{\frac{1}{2}}$ will be the operator whose square coincides with the usual derivative, i.e. $\left(\left(\frac{d}{d x}\right)^{\frac{1}{2}}\right)^{2}(f)=f_{x}$. Furthermore, there are several ways of introducing the fractional derivative, such as Riemann-Liouville or Caputo fractional derivatives. See also [24] for the recent Atangana-Baleanu derivative and related properties [25]. In contrast to Riemann-Liouville's, Caputo fractional derivative allows the resolution of differential equations without having fractional initial conditions. Further information on fractional calculus can be found in [26, 27, 28, 29, 30].

\section{$3 \quad$ Fractional calculus in optics}

Lambert-Beer law states that the evolution of the downward irradiance at the depth $x$ at any wavelength $\lambda$, namely $I_{d}(x, \lambda)$, can be described by

$$
\left\{\begin{array}{l}
\frac{d}{d x} I_{d}(\lambda, x)=\bar{K}_{d}(\lambda) I_{d}(\lambda, x), \quad x>0 \\
I_{d}(\lambda, 0) \in \mathbb{R}^{+}
\end{array}\right.
$$

being $\bar{K}_{d}(\lambda)$ the corresponding spectrally related downward irradiance attenuation coefficient, which can in turn be obtained by calculating inherent optical properties of each organism [31], i. e. measuring chlorophyllspecific absorption and scattering spectra at different mean acclimation irradiance values, when we deal with photosynthetic microorganism cultures.

Furthermore, in [32] deduction of the Lambert-Beer law was explained by means of stochastic approach and linked with Poisson statistics. Later a generalised Lambert-Beer law through fractional calculus was postulated [21]. These results could have been first interpreted as local analysis [33], however it was shown that the domain of validity is broader [34]. In fact, the same result can be obtained when replacing the ordinary derivative by the fractional derivative in Beer-Lambert law (5), that is

$$
\left\{\begin{array}{l}
\left(\frac{d}{d x}\right)^{\alpha} I_{d}(\lambda, x)=\bar{K}_{d}(\lambda) I_{d}(\lambda, x), \quad x>0 \\
I_{d}(\lambda, 0) \in \mathbb{R}^{+} \text {given, }
\end{array}\right.
$$

whose solution is given by

$$
I_{d}(\lambda, x)=E_{\alpha}\left(\bar{K}_{d}(\lambda) x^{\alpha}\right) I_{d}(\lambda, 0)
$$

In this way, a nonlocal analysis is introduced in the Lambert-Beer law, incorporating a tuning parameter $\alpha>0$ that represents the memory in $x$ of the model, which is absent in equation (5). Observe that for mathematical consistence we must restrict the value of $\alpha$ between 0 and 1 , due to the number $(=1)$ of the initial conditions given in (5). In our approach, we have used expression (6) to properly model light attenuation in biological suspensions. To do so, Synechocystis experimental attenuation profiles for different light sources have been estimated by fitting the parameter $\alpha$ of the Mittag-Leffler function. It is 
critical to note that Mittag-Leffler functions do own the necessary properties to correctly predict remaining irradiance within the biological culture as they display power-law decay at further distances but alternatively coincide with the exponential function at closer ones. Though one could expect a two parameters function perform better than a single one, yet other two parameters functions such as the Gamma family do not fulfil the mentioned requirements and hence fail to satisfactorily model the data. This fact together with the mathematical justification of the generalised Lambert-Beer law through Mittag-Leffler functions [21], supports ML functions as the right candidate for modelling light fields within photosynthetic microorganism cultures and equivalent physical systems.

\subsection{Data for modelling assessment}

The data set to be benchmarked with computational results corresponds to Synechocystis cultures exposed to light kept inside a cylindrical vessel. This container has radiometric sensors every $11 \mathrm{~mm}$ to measure PAR downward irradiance within the biological suspension. In this regard, the Synechocystis attenuation data set [8] is composed of light intensity values at increasing depths for the strains here studied (wild-type and Olive) plus two extra antenna mutants (not assessed in this manuscript as we do not own inherent optical properties of those strains), all of them exposed to white LED light at 5 different cell-densities. Such concentrations are given as optical density values $(\mathrm{OD})$ at $750 \mathrm{~nm}$, namely these $\mathrm{OD}_{750}$ values correspond to $0.1,0.5,1.0,2.5$ and 5.0 units.

Moreover, in order to benchmark modelling predictions with the described experimental data, the PAR downward attenuation coefficient $\bar{K}_{d}(P A R)$ needs to be first calculated from the spectrally dependent one $\bar{K}_{d}(\lambda)$. To this purpose, measured inherent optical properties altogether with the depth of the photobioreactor, the cell-density and also the intensity and emission spectrum of the light source have to be taken into account. Following this strategy, classical Lambert-Beer law predictions are available as described in a previous modelling work [3]. In the present manuscript, such modelling framework has been upgraded with Mittag-Leffler functions to improve its predictability capacity.

\section{Results}

In Figure 1, the light field as PAR irradiance value vs. the distance to the illumination source in wild-type (WT) cultures is plotted. It can be seen that in WT for optical densities equal or greater than 1.0, the light field starts to deviate from the pure exponential case, whereas in Olive this happens for optical densities of at least 2.5 (Figure 2). The lowest measured light intensity from the original source within the WT cultures was $1.24 \mu \mathrm{mol}$ photons $\cdot \mathrm{m}^{-2} \cdot \mathrm{s}^{-1}$ at $3.3 \mathrm{~mm}$, whereas for Olive cultures it was 1.60 at $4.4 \mathrm{~mm}$ (both at the most concentrated cell-density). For these points, light attenuation is far from Lambert-Beer law prediction (a line in the semi-logarithmic plot). Indeed, the obtained values for exponential decay are just $8.1 \%(0.1$ $\mu \mathrm{mol}$ photons $\left.\cdot \mathrm{m}^{-2} \cdot \mathrm{s}^{-1}\right)$ and $19 \%\left(0.3 \mu \mathrm{mol}\right.$ photons $\left.\cdot \mathrm{m}^{-2} \cdot \mathrm{s}^{-1}\right)$ of the respectively experimental values in each strain, which leads to the conclusion that in culture regions where practically all the light has been captured by the cells, the Lambert-Beer law does not satisfactorily represent the remaining light field, at least in terms of PAR range integrated values (dashed lines). In fact, this happens in both strains below the euphotic zone, which in our case roughly corresponds to data below $20 \mu$ mol photons $\cdot \mathrm{m}^{-2} \cdot \mathrm{s}^{-1}$.

Alternatively, Mittag-Leffler model (solid lines) is able to correctly predict the remaining downward radiance in both strains at any cell density by fitting the ML $\alpha$ parameter to 0.995 .

It is also remarkable that for the case of cultures exposed to a lamp spectrum with a very narrow emission band, the attenuation is purely exponential in the measured conditions (data not shown [8]). This is due to the fact that monochromatic light is taken up by cells at a constant rate given by the attenuation coefficient value at the peak wavelength of the LED emission spectrum (Gaussian-shaped), whereas in the case of white LED light exposure, attenuation varies gradually: cells mainly absorb light at wavelengths close to the peaks corresponding to the pigments present in them. This selective absorption leads to irradiance diminution of the primarily absorbed wavelengths while the less absorbed ones contribute to the remaining light field at 


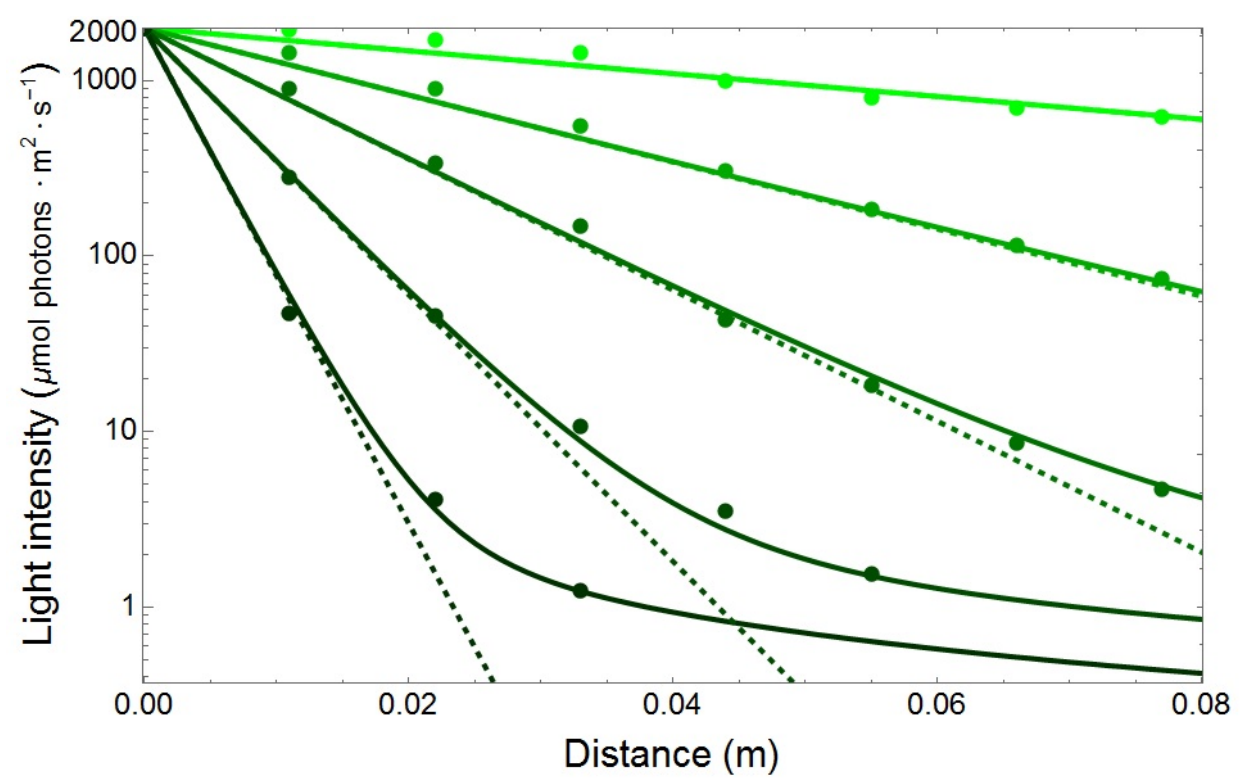

Figure 1: Light attenuation profiles of Synechocystis WT strain cultures exposed to 2000 $\mu \mathrm{mol}$ photons $\cdot \mathrm{m}^{-2} \cdot \mathrm{s}^{-1}$ of white LED light at five different $\mathrm{OD}_{750}$ concentrations $(0.1,0.5,1.0,2.5$ and $5.0)$ are depicted. Dots are the original source samples [8] and lines the modelling outcome (dashed lines Lambert-Beer law, solid lines Mittag-Leffler function). Darker colours correspond to denser suspensions.

deeper distances. In the case of Synechocystis cells, likewise many cyanobacteria which do not possess any photo-active pigment capable of capturing green radiation, the green components of the spectrum are less absorbed, thus light gradually changes from white at the surface to a greener colour at the furthest depth. This leads to a progressively lower attenuation, as cells can hardly capture green light, which explains why a purely exponential decay is not valid in such selectively absorbing media.

\section{Conclusions}

In this work we propose a new scheme to model light attenuation within photosynthetic cultures based on the use of Mittag-Leffler functions. Our scheme is able to substantially improve the estimation of how light intensity decreases at culture depths near or within the euphotic zone, where intensity has already decreased substantially and its spectrum has considerably changed due to selective absorption in the medium. Remarkably, the value of the fitting parameter $\alpha=0.995$ that allows for a proper description of light attenuation within the cultures is exactly the same for both experimental sets corresponding to two different Synechocystis strains. This is not a surprising outcome as both strains have almost the same size, display similar pigment concentration and internal arrangement, except for the missing phycocyanin chromophore in the mutant strain. In view of our results, it may be hypothesised that properly tuned Mittag-Leffler functions are able to represent the long range effects due to selective absorption phenomena in photosynthetic cultures. Indeed, this is the first work where experimental data corresponding to a system with a slowerthan-exponential light decay have been benchmarked with in silico results. By updating the previous exponential-type published model [3] with Mittag-Leffler functions, accurate results are obtained. In fact, this framework generalisation allows the estimation of the light field at any cell density and optical depth inside a PBR for photosynthetic microorganism cultures acclimated to a given irradiance.

As a further work it would be interesting to test our scheme with other cyanobacterial or micro-algal species and to explain, via an analytic estimation, the value of the hyper-parameter taking into account PBR, light, media, and culture characteristics. Furthermore it would be useful to work with a model that can represent the behaviour of the fractional Lambert-Beer law in the case that the parameter varies between 


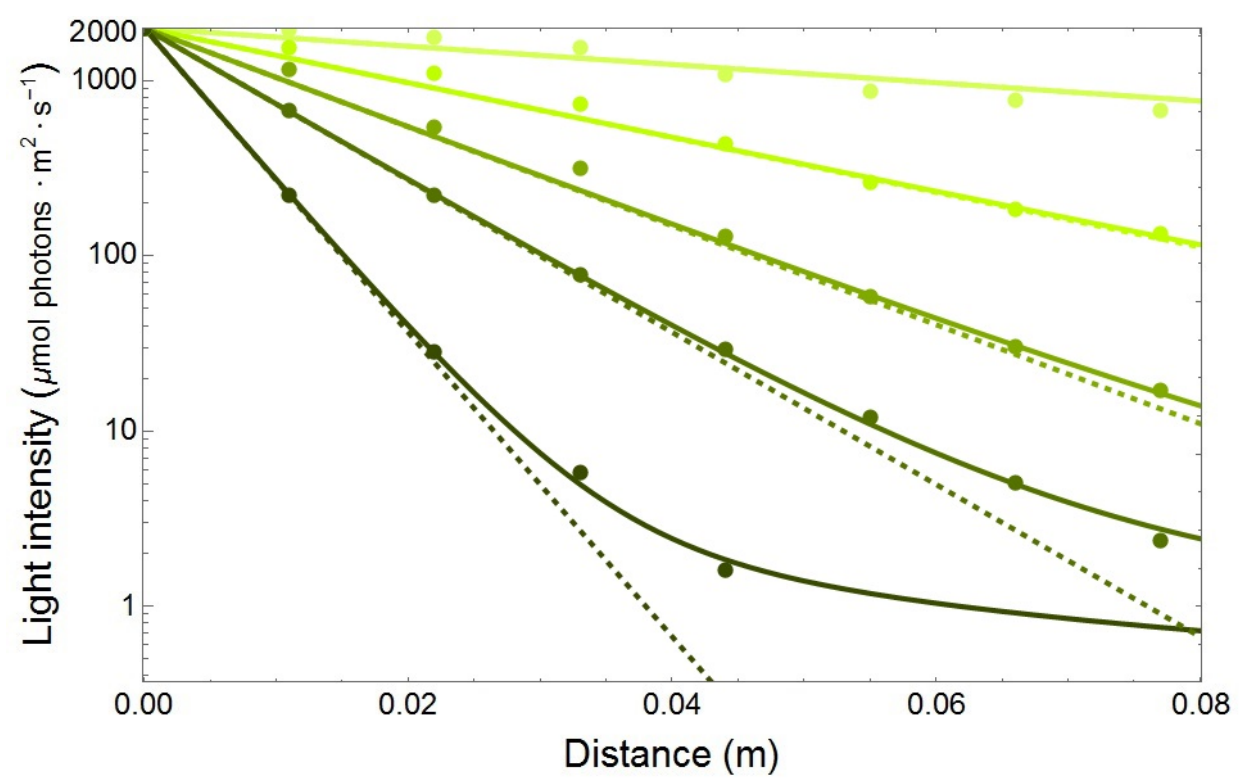

Figure 2: Light attenuation profiles of Synechocystis Olive strain cultures exposed to 2000 $\mu \mathrm{mol}$ photons $\cdot \mathrm{m}^{-2} \cdot \mathrm{s}^{-1}$ of white LED light at five different $\mathrm{OD}_{750}$ concentrations $(0.1,0.5,1.0,2.5$ and 5.0) are depicted. Dots are the original source samples [8] and lines the modelling outcome (dashed lines Lambert-Beer law, solid lines Mittag-Leffler function). Darker colours correspond to denser suspensions.

1 and 2 in a different biological phenomenon. This corresponds to the situation where there are two initial conditions instead of only one as in the classical model (5).

\section{Acknowledgements}

This project has received funding from the European Union's Seventh Programme for research, technological development and demonstration under grant agreement No 308518 CyanoFactory. David Fuente is supported by grant Contratos Predoctorales FPI 2013 of the Universitat Politècnica de València. Carlos Lizama is supported by Programa de Apoyo a la Investigación y Desarrollo (PAID-02-15) de la Universitat Politècnica de València and CONICYT - PIA - Anillo ACT1416. 


\section{References}

[1] M. I. Mishchenko, Electromagnetic scattering by particles and particle groups: an introduction. Cambridge University Press, 2014.

[2] R. W. Preisendorfer, "Application of radiative transfer theory to light measurements in the sea," International Union of Geodesy and Geophysics Monograph, vol. 10, pp. 11-30, 1961.

[3] D. Fuente, J. Keller, J. A. Conejero, M. Rögner, S. Rexroth, and J. F. Urchueguía, "Light distribution and spectral composition within cultures of micro-algae: Quantitative modelling of the light field in photobioreactors," Algal Research, vol. 23, pp. 166 - 177, 2017.

[4] M. I. Mishchenko, "Directional radiometry and radiative transfer: The convoluted path from centuriesold phenomenology to physical optics," Journal of Quantitative Spectroscopy and Radiative Transfer, vol. 146, pp. 4 - 33, 2014. Electromagnetic and Light Scattering by Nonspherical Particles XIV.

[5] J. T. O. Kirk, "The vertical attenuation of irradiance as a function of the optical properties of the water," Limnology and Oceanography, vol. 48, no. 1, pp. 9-17, 2003.

[6] J. T. O. Kirk, "Use of a quanta meter to measure attenuation and underwater reflectance of photosynthetically active radiation in some inland and coastal south-eastern Australian waters," Australian Journal of Marine and Freshwater Research, vol. 28, no. 1, pp. 9-21, 1977.

[7] J. T. O. Kirk, Light and photosynthesis in aquatic ecosystems. Cambridge UK, Cambridge University Press, 1994.

[8] D. J. Lea-Smith, P. Bombelli, J. S. Dennis, S. A. Scott, A. G. Smith, and C. J. Howe, "Phycobilisomedeficient strains of Synechocystis sp. PCC 6803 have reduced size and require carbon-limiting conditions to exhibit enhanced productivity," Plant Physiology, vol. 165, no. 2, pp. 705-714, 2014.

[9] H. Schiessel, R. Metzler, A. Blumen, and T. F. Nonnenmacher, "Generalized viscoelastic models: their fractional equations with solutions," Journal of Physics A: Mathematical and General, vol. 28, no. 23, pp. 6567-6584, 1995.

[10] S. F. Zaman, D. Baleanu, and I. Petráš, "Measurement of para-xylene diffusivity in zeolites and analyzing desorption curves using the mittag-leffler function," Fractional Calculus and Applied Analysis, vol. 19, no. 2, pp. 551-560, 2016.

[11] R. Metzler and J. Klafter, "The random walk's guide to anomalous diffusion: a fractional dynamics approach," Physics Reports, vol. 339, no. 1, pp. 1-77, 2000.

[12] W. G. Glöckle and T. F. Nonnenmacher, "A fractional calculus approach to self-similar protein dynamics," Biophysical Journal, vol. 68, no. 1, pp. 46-53, 1995.

[13] A. E. Calık, H. Sirin, H. Ertik, and M. Sen, "Analysis of charge variation in fractional order LC electrical circuit," Revista Mexicana de Física, vol. 62, no. 5, pp. 437-441, 2016.

[14] M. N. Berberan-Santos, "Properties of the Mittag-Leffler relaxation function," Journal of Mathematical Chemistry, vol. 38, no. 4, pp. 629-635, 2005.

[15] H. J. Haubold, A. M. Mathai, and R. K. Saxena, "Mittag-Leffler functions and their applications," Journal of Applied Mathematics, vol. ID 298628, 2011.

[16] F. Mainardi, R. Gorenflo, and A. Vivoli, "Renewal processes of Mittag-Leffler and Wright type," Fractional Calculus and Applied Analysis, vol. 8, no. 1, pp. 7-38, 2005. 
[17] R. Hilfer, "Fractional diffusion based on Riemann-Liouville fractional derivatives," The Journal of Physical Chemistry B, vol. 104, no. 16, pp. 3914-3917, 2000.

[18] K. R. Lang, Astrophysical Formulae-Volume I: Radiation, Gas Processes and High Energy Astrophysics. Springer, 1999.

[19] R. K. Saxena, A. M. Mathai, and H. J. Haubold, "On fractional kinetic equations," Astrophysics and Space Science, vol. 282, no. 1, pp. 281-287, 2002.

[20] L. Preda, "Splitting and accelerating gaussian beam modulated by Mittag Leffler function," OptikInternational Journal for Light and Electron Optics, vol. 127, no. 3, pp. 1066-1070, 2016.

[21] G. Casasanta, D. Ciani, and R. Garra, "Non-exponential extinction of radiation by fractional calculus modelling," Journal of Quantitative Spectroscopy and Radiative Transfer, vol. 113, no. 2, pp. 194-197, 2012.

[22] V. Tramontana, G. Casasanta, R. Garra, and A. M. Iannarelli, "An application of Wright functions to the photon propagation," Journal of Quantitative Spectroscopy and Radiative Transfer, vol. 124, pp. $45-48,2013$.

[23] R. Ibrahim and C. Ozel, "On multi-order fractional differential operators in the unit disk," Filomat, vol. 30, no. 1, pp. 73-81, 2016.

[24] A. Atangana and D. Baleanu, "New fractional derivatives with nonlocal and non-singular kernel: Theory and application to heat transfer model thermal science," Thermal Science, p. 18, 2016.

[25] T. Abdeljawad and D. Baleanu, "Integration by parts and its applications of a new nonlocal fractional derivative with mittag-leffler nonsingular kernel," Journal of Nonlinear Sciences and Applications, vol. 10, p. 1098-1107, 2017.

[26] I. Podlubny, Fractional differential equations: An introduction to fractional derivatives, fractional differential equations, to methods of their solution and some of their applications, vol. 198. Academic Press, 1998.

[27] M. D. Ortigueira, Fractional Calculus for Scientists and Engineers, vol. 84. Springer Science \& Business Media, 2011.

[28] D. Baleanu, J. A. T. Machado, and A. C. J. Luo, Fractional Dynamics and Control. Springer Science \& Business Media, 2011.

[29] D. Baleanu, K. Diethelm, E. Scalas, and J. J. Trujillo, Fractional Calculus: Models and Numerical Methods. World Scientific, 2016.

[30] T. Abdeljawad and D. Baleanu, "Monotonicity results for fractional difference operators with discrete exponential kernels," Advances in Difference Equations, vol. 2017, no. 1, p. 78, 2017.

[31] J. T. O. Kirk, "Attenuation of solar radiation in scattering-absorbing waters: a simplified procedure for its calculation," Applied Optics, vol. 23, pp. 3737-3739, 1984.

[32] A. B. Kostinski, "On the extinction of radiation by a homogeneous but spatially correlated random medium," Journal of the Optical Society of America A, vol. 18, no. 8, pp. 1929-1933, 2001.

[33] A. Borovoi, "On the extinction of radiation by a homogeneous but spatially correlated random medium: comment," Journal of the Optical Society of America A, vol. 19, no. 12, pp. 2517-2520, 2002.

[34] A. B. Kostinski, "On the extinction of radiation by a homogeneous but spatially correlated random medium: reply to comment," Journal of the Optical Society of America A, vol. 19, no. 12, pp. 2521-2525, 2002. 